\title{
Evaluating Possible Methods and Approaches for Registering of Electromagnetic Waves Emitted from the Human Body
}

\author{
Ignat Ignatov ${ }^{1 *}$ Oleg Mosin ${ }^{2}$ Hugo Niggli ${ }^{3}$ Christos Drossinakis ${ }^{4}$ \\ 1. DSc, professor, Scientific Research Center of Medical Biophysics (SRCMB), \\ 32 N. Kopernik St., Sofia 1111, Bulgaria
}

2. PhD, Biotechnology Department, Moscow State University of Applied Biotechnology, 33 Talalikhina St., Moscow 109316, Russian Federation

3. DSc, BioFoton AG 27 Route d`Essert., 1733 Treyvaux, Switzerland

3. Dipl. Eng., IAWG-GmbH, 61A Königsteiner St., Frankfurt, Germany, Chalkida, Greece

* E-mail of the corresponding author: mbioph@ dir.bg

\begin{abstract}
This paper presents the results of evaluation of possible biophysical methods and approaches for registering various non-ionizing radiation (NIR) wave types of the human body in the electromagnetic range. Many types of NIR (electromagnetic waves, infrared radiation, thermo radiation, bioluminescence) emitted from the human body were reviewed. In particular the results of spontaneous biophoton emission and delayed luminescence from the human body are submitted along with infrared thermography (IRT) results. It was shown that $1 \mathrm{~cm}^{2}$ of skin generally emits $\sim 85$ photones for $1 \mathrm{~s}$. The intensity of biophoton emission ranges from $10^{-19}$ to $10^{-16} \mathrm{~W} / \mathrm{cm}^{2}$ (approx. 1-1000 photons $\mathrm{cm}^{-2} \cdot \mathrm{s}^{-1}$ ). The specific photon emission from part of the human thumb was detected as a spectrum of various colours with the method of Color coronal spectral analysis on a device with an electrode made of polyethylene terephthalate (PET hostafan) with applied electric voltage of $15 \mathrm{kV}$, electric impulse duration $10 \mu \mathrm{s}$, and electric current frequency $15 \mathrm{kHz}$. It was established that photons corresponding to a red color emission of the visible electromagnetic spectrum have energy at $1.82 \mathrm{eV}$. The orange color of the visible electromagnetic spectrum has energy at 2.05 , yellow -2.14 , blue-green (cyan) -2.43 , blue -2.64 , and violet $3.03 \mathrm{eV}$. The reliable result measurement norm was at $\mathrm{E} \geq 2.53 \mathrm{eV}$, while the spectral range of the emission was within 380-495 $\mathrm{nm}$ and 570-750 $\mathrm{nm} \pm 5 \mathrm{~nm}$. Some important physical characteristics were also demonstrated (energy of hydrogen bonds, wetting angle, surface tension) of water by the methods of non-equilibrium energy (NES) and differential non-equilibrium energy (DNES) spectrum of water, that helps understand in general how electromagnetic radiation interacts with water and establishes the structural alterations of water.
\end{abstract}

Keywords: electromagnetic waves, infrared radiation, thermo radiation, bioluminescence, color coronal spectral analysis, NES, DNES

\section{Introduction}

All living organisms have a cellular, and therefore, a molecular organized structure. The living processes inside them run on a cellular and a molecular level. Bioelectrical activity is one of the very important physical parameters of living organisms (Ignatov et al., 1998). Bioelectric potentials generated by various cells are widely used in medical diagnostics and are recorded as electrocardiogram, electromyogram, electroencephalogram, etc. It was proved that the human body and tissues emanate weak electromagnetic waves, the electric voltage of which is denoted as resting potential, action potential, omega-potential etc. (Dobrin et al., 1979; Adey, 1981). Between the outer surface of the cell membrane and the inner contents of the cell there is always the electric potential difference, which is created because of different concentrations of $\mathrm{K}^{+}, \mathrm{Na}^{+}$and $\mathrm{Cl}^{-}$inside and outside of the cell and their different permeability through the cell membrane (Kiang et al., 2005). Their value in the human body varries $\sim 50-80 \mathrm{mV}$, and is defined by the galvanic contact of a voltmeter input with an object that indicates the galvanic type of their source (Cleary, 1993). When excited, a living cell changes the membrane electric potential due to changes in membrane permeability and active ion movement through the membrane. In cells of excitable tissues (muscle, nervous), these processes can occur within a very short time intervals (milliseconds) and are called "current action" potential. Their magnitude makes up 120 mV. Electromagnetic fields refer to non-ionizing radiation (NIR), e.g. the radiative energy that, instead of producing charged ions when passing 
through matter, has sufficient energy only for excitation. Nevertheless it is known to cause biological effects (Kwan-Hoong, 2003). The NIR spectrum is divided into two main regions, optical radiations and electromagnetic fields. The optical spectrum can be further sub-divided into ultraviolet, visible, and infra-red. The electromagnetic fields are further divided into radiofrequency (microwave, very high frequency and low frequency radio wave). NIR encompasses the long wavelength $(>100 \mathrm{~nm})$ and low photon energy $(<12.4 \mathrm{eV})$ portion of the electromagnetic spectrum, from $1 \mathrm{~Hz}$ to $3 \cdot 10^{15} \mathrm{~Hz}$. Research carried out in the 1990ies and subsequent years established the property of animal and plant tissues to generate relatively strong transient NIR electric fields due to mechanical stresses and temperature changes in biological structure (Anderson, 1993). These electric fields are mainly due to the piezoelectric and pyroelectric voltage electric polarization of natural biological structures. Owing to cell metabolism, electric dipoles (polar and ionized molecules) involved in polarization of biostructures are continuously destroyed and restored, i.e. this is a non-equilibrium polarization (Barnes \& Greenebaum, 2006). Such type of non-equilibrium electric polarization is known as a main characteristic of electrets (Gubkin, 1978). Electrets include dielectric insulators and semiconductors, which, under certain conditions, e.g. under the influence of a strong electrostatic field or ionizing radiation, light and other factors, acquire the property to generate an external electric field, existing for a long time (days, years) and slowly diminish due to the destruction of their substance by polarization (Sessler \& Gerhard-Multhaupt, 1998). Along with the electromagnetic field, electrets generate specific elecric currents produced by heating - thermally stimulated current (TSC) (Gross, 1964). Electrets belonging to the non-galvanic type of electrical sources tend to a strong electric field (up to $10^{6} \mathrm{~V} / \mathrm{m}$ ) and to the infinitesimal electric current $\left(\sim 10^{-14} \mathrm{~A} / \mathrm{mm}^{2}\right)$. By analogy with the physical fields, the electric field emitted from the human body and its physical alterations resemble the electric field generated by electrets. The electrets play an important role in the functioning of many biological structures as they themselves possess electret properties. The bioelectret fields registered on the surface of the human body are basically generated by the basal cells of the epidermis (Marino, 1988). Dermis cells adjacent to the bottom layer of basal cells are surrounded by a conductive interstitial fluid whose electric voltage while grounding on the human body is close to zero (so called ground potential). This interstitial fluid screens off electromagnetic fields of underlying tissues. With the average thickness of the epidermis $(\sim 0.1 \mathrm{~mm})$ and the maximum value of electric voltage $(\sim 30.0 \mathrm{~V})$, the electric field strength can reach significant values at $\sim 300000$ $\mathrm{V} / \mathrm{m}$ (Seto et al., 1992). The strength of the electric field is quite sufficient for its influence on the biological processes in cells and surrounding tissues, including the synthesis of proteins and nucleic acids (Liboff at al., 1984; Frey, 1993; Shimizu et al., 1995). This electric field along with the field of transmembrane assymetry of ions concentrated at the inside and outside of the membrane $\left(\sim 10^{5} \mathrm{~V} / \mathrm{cm}^{2}\right)$ can participate in the cooperative effects in cell membrane structures (Holzel \& Lamprecht, 1994; Miller, 1986). Thus, owing to the bioelectret condition of certain subcellular structures in the cell and its surroundings a slowly oscillating electric field is generated that is strong enough to influence the biological processes. This field and the electric field due to the piezoelectric voltage and intramembrane electric field forms the total electromagnetic field of the cell and its supracellular structures. It is known that the human skin emanates electromagnetic waves in close ultraviolet range, optic range and also in close infrared range. Infrared thermal bioradiation is found in the middle infrared range at wavelengths from 8 to $14 \mu \mathrm{m}$. At a wavelength of $9.7 \mu \mathrm{m}$ infrared bioradiation has its maximum value at $\mathrm{t}=36.6{ }^{\circ} \mathrm{C}$. At this temperature the skin emission is closest to the emission of an absolute black body (ABB) having the same temperature. Infrared emission penetrates the skin surface at a depth of $\sim 0.1 \mathrm{~mm}$, and is reflected in accordance with the physical laws of reflection of the visible part of the electromagnetic spectrum. Evidently, radiation energy influences tissues while being absorbed by them. Yu.V. Gulyaev and E. E. Godik (Gulyaev \& Godik, 1984) determined that the threshold of skin sensitivity for infrared radiation compiled $\sim 10^{-14}$ $\mathrm{W} / \mathrm{cm}^{2}$. When thermal influence is applied to the point of threshold skin sensitivity, there develops a physiological reaction toward the thermal flow. The intensity of the radiated thermal flow generated by the skin makes up $\sim 2.610^{-2} \mathrm{~W} / \mathrm{cm}^{2}$. The second component of electromagnetic waves is bioluminescence (Young \& Roper, 1976; Chang et al., 1998). It is supposed that biophotons, or ultraweak photon emissions of biological objects, are weak electromagnetic waves in the optical range of the spectrum (Cohen \& Popp, 1997). The typical observed emission of biological tissues in the visible and ultraviolet frequencies ranges from $10^{-19}$ to $10^{-16}$ W/cm ${ }^{2}$ ( 1-1000 photons $\mathrm{cm}^{-2} \mathrm{sec}^{-1}$ ) (Edwards et al., 1989; Choi et al., 2002). This light intensity is much weaker than the one to be seen in the perceptually visible and well-studied spectrum of normal bioluminescence detectable above the background of thermal radiation emitted by tissues at their normal temperature (Niggli, 1993). Bioelectric emission from parts of the human body like thumbs can be easily detected with the method of Color coronal spectral analysis by applying gas electrical discharge of high voltage and frequency developed by I. Ignatov (Ignatov, 2005). Its advantages include safety, sterility, clarity and interpretability of the data obtained, ease of storage and subsequent computer data processing, the ability to monitor the development of processes in 
time, comparing the structural, functional and temporal processes, etc. The purpose of this research was the studying of possible biophysical methods and approaches for registering various NIR wave's types emitted from the human body (electromagnetic waves, infrared radiation, thermo radiation) and methods of their visualization by different techniques including magnetography, infrared thermography, chemiluminescence and coronal gas discharge spectral analysis.

\section{Materials and methods}

\subsection{Infrared thermography (IRT)}

The research was made using infrared thermography (IRT) method according to M. Marinov. The range of the infrared thermal-imaging camera was in the middle infrared range from $9 \mu \mathrm{m}$ to $14 \mu \mathrm{m}$. The temperature range was from $24.0{ }^{0} \mathrm{C}$ to $38.0{ }^{0} \mathrm{C}$. The first camera was Inframetrics/FLIR ThermaCam PM 290 wave type. FLIR ThermaCam PM 290, FLIR 390, Inframetrics PM 250 and Inframetrics PM 350 thermal infrared cameras were of FLIR short wave type, handheld, Focal Plane Array cameras are capable of temperature measurement. These cameras stored images on a PCMCIA Card, and the images were further analyzed using one of several available FLIR software packages (Thermogram 95, FLIR Reporter 2000 Software, Researcher 2000). The second camera (D.I.T.I.) was a totally non-invasive clinical imaging camera for detecting and monitoring a number of diseases and physical injuries, by revealing the thermal abnormalities present in the human body's patterns. It was used as a tool for diagnosis and prognosis, as well as monitoring therapy progress; the type of this device was TB $04 \mathrm{~K}$.

\subsection{Registration of electromagnetic fields}

The registration of electromagnetic fields was used with super conductive detectors based on Joseffson junctions - device made by sandwiching a thin layer of insulating nonsuperconducting material between two layers of superconducting cooper pairs (S-I-S).This allows the registering of magnetic fields $10^{10}$ times weaker than the Earth's magnetic field. The study of electric field emitted by the human body was done using a standard Faraday cage formed by conducting material (aluminum foil) blocks with external static and non-static electric fields by channeling electricity through the conducting material, providing constant voltage on all sides of the enclosure.

\subsection{Biophoton detection}

The measurement was performed with photoemission detector with photomultiplier (EMI9558QA selected type) in a dark room impervious to the light wherein the light was minimized about $\sim 5$ cps. This was 4 times lower than the noise of the cooled photomultiplier $-20 \mathrm{cps}$. The photomultiplier was connected through an amplifier and other intermediate devices to a potentiometer recorder or a personal computer. The detection of biophoton emission was performed within a time interval with real count rate $3 \mathrm{cps}$, for $30 \mathrm{~min}$, with reliability $\mathrm{p}<0.05$. The registration was performed from an area of the skin with a diameter of $7 \mathrm{~cm}$ within a time interval of $100 \mathrm{~ms}$ or $1 \mathrm{~s}$. The calculation of biophoton emission was measured in counts/100 ms. The irradiation time of the 150 W-tungsten lamp was $5 \mathrm{~s}$. Within $100 \mathrm{~ms}$ after switching off the external lamp, the first measurements of delayed luminescence was recorded; then 256 units of the delayed luminescence were recorded. Delayed luminescence of biological objects and tissues in terms of coherent states was detected within time intervals of $100 \mathrm{~ms}$. Relaxation function was of 25.6 units. These dates are object to further scrutinized analysis (Cohen \& Popp, 1997).

\subsection{Color coronal gas discharge spectral analysis}

Experiments were carried out by Selective high-frequency electric discharge (SHFED) on a device with the electrode made of polyethylene terephthalate (PET, hostafan) with an electric voltage on the electrode $15 \mathrm{kV}$, electric impulse duration $10 \mu \mathrm{s}$, and electric current frequency $15 \mathrm{kHz}$. The electrode of the device was made of hostafan, and was filled up with electro-conductive fluid. The spectral range of the emission was in the range 380-495 $\mathrm{nm}$ and 570-750 $\pm 5 \mathrm{~nm}$. The measurements were registered in electronvolts $(\mathrm{eV})$. The authors of the device are I. Ignatov and Ch. Stoyanov. Detection of gas disharge glowing was conducted in a dark room equipped with a red filter. A color film was put on the electrode. The object under study (human thumb) was placed on top of color film. Between the object and the electrode were generated impulses of electric voltage 15 $\mathrm{kV}$ and electric current frequency $-15-24 \mathrm{kHz}$; on the reverse side of the electrode was applied the transparent electrically conductive thin copper coating. Under these conditions in the thin contact gas space between the studied object and electrode was generated gas electric discharge in the form of characteristic glow around the object - a corona gas electric discharge in the range of 280-760 nm illuminates a color photographic film. The parameters of the corona gas discharge glow depend on the bioelectric properties of the studied object. Along 
with the visible range, for this method were obtained color spectra in the ultraviolet (UV) and the infrared (IR) range. Evaluation of the physical and biophysical parameters of snapshots was based on the analysis of images treated by standard software package. Statistical processing of the experimental data was performed using the statistical package STATISTISA 6 using Student's $t$-criterion (at $\mathrm{p}<0.05$ ).

\subsection{NES and DNES experiments on interaction of electromagnetic field with water}

The research was made with the method of Non-equilibrium spectrum (NES) and Differential non-equilibrium spectrum (DNES). The device measures the angle of evaporation of water drops from $72^{0}$ to $0^{\circ}$. As the main estimation criterion was used the average energy $\left(\Delta \mathrm{E}_{\mathrm{H}} \ldots \mathrm{O}\right)$ of hydrogen $\mathrm{O}$...H-bonds between $\mathrm{H}_{2} \mathrm{O}$ molecules in water's samples. The spectrum of water was measured in the range of energy of hydrogen bonds $0.08-0.1387 \mathrm{eV}$ or $8.9-13.8 \mu \mathrm{m}$ with a specially designed computer program.

\section{Results and discussion}

\subsection{Electric fields}

The electric field surrounding the human body with frequency $v=1 \cdot 10^{3} \mathrm{~Hz}$ is created by electrochemical processes in the organism and is modulated by the rhythm of internal organs (Ignatov et al., 1998). The spatial distribution of the electric field around the body reflects the teamwork of the different organs and systems in the organism. There are also electric fields, which are generated by accumulation of triboelectric (caused by friction) charge on the epidermis, which depends on epidermal electric resistance and varies from $10^{9}$ to $10^{11} \Omega / \mathrm{cm}^{2}$. Radiothermal emission is being detected in the centimeter and decimeter range of the spectrum. This type of emission is connected with the temperature and the biorhythms of the internal organs, and is being absorbed by the surface skin layer at a depth from $5 \mathrm{~cm}$ to $10 \mathrm{~cm}$ (Gulyaev \& Godik, 1984). Long persistent electric field emitted from the human body can be detected with an electrometer voltmeter after neutralizing electric charges on the skin caused by triboelectric charges. The electric strength of this field is undergoing slow oscillations, and most patients exert its value within the range of $100-1000 \mathrm{~V} / \mathrm{m}$ at a distance of 5-10 $\mathrm{cm}$ from the body. People in a state of clinical death usually have the electric field strength's value reduced to $10-20 \mathrm{~V} / \mathrm{m}$ after $2-3$ hours of cardiac arrest. Intensity vector of the detected electric field is found to be normal at the surface of the skin, and the electric voltage is inversely proportional to the distance. On the skin surface the electric voltage of the field (the difference of its electric potential with respect to ground potential) reaches essential values of $\sim 10000 \mathrm{mV}$ or more, i.e., is about 1000 times greater than the source electric voltage of the electric unit above the bioelectric potentials. This allows us to characterize the electric field detected from the human body as relatively strong electric field emitted from living tissues. Its electric voltage was measured by electrometric methods, identified by a non-galvanic type of its source. If the physical basis of the generation of a relatively strong electric field in the human tissue is non-equilibrium electric polarization of the substance due to metabolic processes, the electric field strength should depend on these processes. As noted above, this dependence is actually observed: inhibition of tissue metabolism due to hypoxia during cardiac arrest was accompanied by drop in the electric field strength. This relationship is confirmed in experiments on animals (Geraldy et al., 2008). For example, in rats inhibition of metabolism of the tissue due to cardiac arrest (death of the animal) or by general anesthesia is accompanied by a significant drop in the electric field strength (Bars \& Andre, 1976). Electric fields depend on the magnitude of the electric voltage and the distance from the source (Kwan-Hoong, 2003). Generally, the electric voltages are stable and remain the same; however electric fields are easily perturbed and distorted by many surrounding objects. Relatively strong electric field investigated in humans and animals is being formed evidently by the skin's biostructures, since the electric fields of the underlying tissues are largely shielded by conductive interstitial fluid (Goodman et al., 1995; Gulyaev \& Godik, 1990). The basal cells of the epidermis - the top layer of the skin, contribute the most to the detected electric field. Electric polarization vector of these cells is normal to the surface of the skin, i.e., coincides with the electric voltage's vector field, and yet it is inherent in the metabolism intensity, conditioning the generation of the electric field.

\subsection{Magnetic fields}

Magnetic field of a living organism can be caused by three reasons. First of all, it is ion channels arising from the electrical activity of cell membranes (primarily muscle and nervous cells). Another source of magnetic fields are the tiny ferromagnetic particles, trapped or specially introduced into the human body. These two sources create their own magnetic fields. In addition, at imposition of external magnetic field there appears inhomogeneity of the magnetic susceptibility of different organs and tissues distorting the external magnetic field (Wikswo \& 
Barach, 1980). The magnetic field in the last two cases is not accompanied by the appearance of the electric field, so the study of the behavior of magnetic particles in the human body and the magnetic properties of various organs are applicable only with magnetometric methods. Biocurrents, on the contrary, except for the magnetic fields, create the distribution of electric potentials on a body's surface. Registration of these electric potentials has long been used in research and clinical diagnostics - in electrocardiography, electroencephalography, etc (Cohen, 1968). It would seem that their magnetic counterparts, i.e. magnetocardiography and magnetoencephalography recording the signals from the same electrical processes in the body, will give almost the same information about the studied organs. However, as follows from the theory of electromagnetism, the structure of the electric current source in the electric conductive medium (the body) and the heterogeneity of the medium have significantly different impact on the distribution of magnetic and electric fields: some types of bioelectric activity manifest themselves primarily in the electric field, giving a weak magnetic signal, while others - on the contrary, create a rather strong magnetic signal (Zhadin, 2001; Anosov \& Trukhan, 2003). Therefore, there are many biophysical processes whose observation is preferable by magnetographic methods. Magnetography does not require direct contact with the investigated object, i.e., it allows carrying out measurements over a bandage or other obstructions. It is not only practically useful for diagnostics, but has a fundamental advantage over electrical methods towards data recording, as the attachment of the electrodes on the skin can be a source of slowly varying contact electric potentials. There are no such spurious noises while using magnetographic methods, therefore, magnetography allows, in particular, to reliably explore slowly occurring processes (with the characteristic time of tens of minutes). Magnetic fields rapidly diminish with distance from the source of the activity, as they are caused by relatively strong currents running in the body, while the surface potentials are determined mainly by the weaker and "smeared" electric currents in the skin. Therefore, magnetography is more convenient for accurate determination (localization) of bioelectric activity parts on the human body. And finally, the magnetic field vector is characterized not only by the absolute value but also by the direction, which also may provide additional useful information. However, it should not be assumed that the electricity and magnetographic methods compete with each other. On the contrary, it is their combination that gives the most complete information about the processes being investigated. But for each of the individual methods, there are practical areas wherein the use of any one of them is preferable. Water is the main substance of all living organisms and the magnetic field exerts a certain influence on water. This influence is a complex multivariate influence, which the magnetic field exerts on dissolved in water metal cations $\left(\mathrm{Fe}^{2+}, \mathrm{Fe}^{3+}\right)$ and the structure of the hydrates and water associates (Mosin, 2011). It was experimentally proved that the magnetic field acts much weaker on still unmoved water, because water has a conductivity; as water moves in the electromagnetic field, a small electric current is generated (Mosin, 2012). The research performed with superconductive detectors based on Joseffson junctions shows that magnetic fields around the human body are in the range from 1 to $100 \mathrm{~Hz}$. The magnetic activity of the brain for example makes up $\sim 3010^{15} \mathrm{~T} / \mathrm{Hz}^{1 / 2}$. The magnetometric system has a sensitivity of $10 \cdot 10^{15} \mathrm{~T} / \mathrm{Hz}^{1 / 2}$ in the range of 1 to $100 \mathrm{~Hz}$ (Gulyaev \& Godik, 1990).

\subsection{Chemiluminescence}

Chemiluminescence denotes luminescence accompanying chemical reactions, detected in the near infrared, the optical and the near UV-range of the electromagnetic spectrum. When chemiluminescence takes place in living organisms, the phenomenon is called bioluminescence. The term is generally used for higher luminance ATP-dependent luciferin/luciferase systems observed in Lampyridae lightning bugs (Rauhut, 1985). Although there are bacterial, latia and dinoflagellate luciferin, and coelenterazine found in some bacteria, freshwater snails, dinoflagellates, radiolarians, shrimp, squid and deep-sea fish species (Hastings, 1983). Chemiluminescence is observed in reactions accompanied by allocation of large amounts of energy, such as the reaction of combining two radicals, or in reactions involving peroxides, e.g. peroxide oxidation of lipids (Halliwell \& Gutteridge, 1989). Peculiar ("ultra-weak") glow of cells and tissues of animals and mammals is caused by free radical reactions: lipid radicals and oxygen, and nitrogen oxides - compounds that play an essential role in cell metabolism, and under certain conditions in the development of a number of pathological conditions. It is suggested that the major radicals responsible for the light emission are excited triplet carbonyl and excited singlet oxygen, and that these radicals arise through the decomposition of hydroperoxides formed in the process of lipid oxidation (Zlatkevich \& Kamal-Eldin, 2005; Porter \& Wujek, 1988). The process of lipid peroxidation (LPO) is an important cause of the accumulation of cellular defects caused by radicals. LPO main substrate is polyunsaturated fatty acids in composition of cell membranes and lipoproteins. Their attack by oxygen radicals leads to the formation of hydrophobic radicals, interacting with each other (Vladimirov, 1996). Many different mechanisms have been suggested for the oxidative lipid fragmentation that produces biologically active aldehydes as 4-hydroxynon-2-enal (HNE), oxononanoyl phosphatidylcholine (ON-PC) from linoleic acid (LA) esters, or HNE and oxovaleroyl phosphatidylcholine (OV-PC) (Esterbauer et al., 1990). Processes of life are 
almost always accompanied by a very weak radiation, which is sometimes called ultra-low illumination or radiation of cells and tissues (Boveris et al., 1980). Some organisms possess the ability to emit bright light at photon fluxes below about $10^{4}$ photons $\mathrm{cm}^{-2} \mathrm{~s}^{-1}$, visible to the naked eye, this phenomenon is denoted "bioluminescence". In biochemical systems, i.e. in tissue's homogenates, cell suspensions or cell organelles, mixtures of enzymes and substrates, chemiluminescence in most cases has an extremely low intensity, and requires particularly sensitive equipment for its detection and measurement (Popp et al., 2002). Some substances - enhancers, have the ability to essentially enhance the chemiluminescence, sometimes many thousands of times (activated, or enhanced chemiluminescence). In addition, weak luminescence is accompanied by the formation of free radicals under the action of a number of physical factors on the object: at ionizing radiation, radiochimoluminescence is observed, at ultraviolet or visible light illumination - photochimoluminescence, at passing an electric current - electroluminescence, with ultrasound - sonoluminescence, under the influence of friction forces - triboluminescence. Chemiluminescence differs from fluorescence in that the electronic excited state is derived from the product of a chemical reaction rather than the more typical way of creating electronic excited states, namely adsorption. It photomemical reactions, in which light is used to drive an endothermic chemical reaction, light is generated from a chemically exothermic reaction. At present time, quite a lot of chemical reactions are known involving the formation of luminescence glow. In most cases they are generally quite complex processes having many intermediate stages, but the basic processes leading to luminescence glowing in general are similar. They include the separation and transfer of charged particles (electrons and free radicals), the electron transfer (redox reactions) at one of the higher energy levels with the formation of the reaction product in an electron-excited state and further releasing of a photon in the transition of the molecule to the low excited electronic ground state with a lower energy level (luminescence). Theoretically, in this process on each molecule of the reactant one photon should be allocated. Chemiluminescence accompanies many chemical reactions (ozonation and fluorination reactions, the oxidation of phosphorus and complex organic substances, lipids) and has an impulse mode; the signals of this process usually are very weak. Thus, the human skin dissociates few photons per $1 \mathrm{sec}$. with emission power level $\sim 10 \mathrm{~mW} / \mathrm{cm}^{2}$ (Gulyaev, Godik, 1991).

Luminescence of cells and tissues is accompanied by three types of reactions:

- $\quad$ Reactions with active oxygen;

- Chain reactions of lipid peroxidation;

- $\quad$ Reactions involving nitric oxide (NO).

The more lipid radicals the system contains, e.g. the more energetic the chain reaction of lipid oxidation, the higher the intensity of chemiluminescence accompanying the reaction of radicals. Substances reacting with free radicals and thereby inhibiting the chain lipid oxidation (so-called antioxidants) simultaneously inhibit chemiluminescence. That inhibition of chemiluminescence by cells and tissues by such antioxidants as tocopherol (vitamin E), indicates that chemiluminescence is stipulated by lipid oxidation chain reactions. On the other hand, studying the impact of various natural and synthetic compounds on the time (kinetics) of chemiluminescence, one can judge on the ability of these substances to protect our body from the harmful effects of free radicals and thereby select candidates to certain medications. Chemiluminescent methods are used for recording ultra-weak light waves accompanying the chemical and biochemical reactions involving the formation of free radicals. They do not require special laboratory conditions and special material preparation for analysis, they are characterized by high sensitivity, reliability, and meet the requirements for rapid methods of express research. Chemiluminescent methods are widely used in biomedical diagnostics for studying the molecular basis of physiological processes in biological systems and general mechanisms of development of pathological conditions.

\subsection{Biophoton emission}

The term biophoton emission means a photon of non-thermal origin spontaneous emission in the visible and UV-spectrum emitted from all biological objects and tissues and covered over a wide range of wavelengths, from 200 to $800 \mathrm{~nm}$. Biophoton emission was discovered by the biologist Alexander Gurwitsch (USSR) who was among the first to discover that in the process of cell mitogenesis, cells emitted ultra-weak UV waves, named mitogenetic or Gurwitsch rays (Gurwitsch, 1959; Beloussov et al., 1997). The biophoton emission is evidently linked to the endogenous production of excited states within the biological system. As a possible carrier of biophotone emission A. Gurvitsh offered chromatin - the complex of DNA with special proteins that during eukaryotic cell division form chromosomes - thread-like structures inside the cell's nucleus, consisting of DNA, RNA and proteins. Cells that do not contain chromatin, do not possess the ability to emit biophotones. It was found that if the other surrounding cells were under mitogenetic rays, the mitosis increased, i.e. it stimulated the cell growth (Gurwitsch, 1988; Nikolaev, 2000). It was also demonstrated that mitogenetic radiation can not only 
stimulate, but also inhibit the cell growth. Further, F.A. Popp developed a biophoton theory to explain their possible biological role and the ways in which they may control biochemical processes, growth, cell differentiation etc. (Popp, 2005). This work has been further corroborated by the Italian biophotonic group of Musumeci and co-authors (Niggli et al., 2005, Scordino et al., 2014). In cellular systems, this low level radiation has been measured by various scientific researchers worldwide and was reviewed most recently by Niggli (2014 a,b). For many years the existence of Gurwitsch rays has been uncertain because of the very contradictory experimental results for their detection. The situation has changed for the better with the development of more sensitive resolution technical devices for their precise detection (Inaba, 1988; Motohiro, 2004). Contemporary photon-emission detectors used for photon's detection are divided into two classes: photodetectors, or photon detectors and temperature detectors. In photodetectors photons absorbed by the material of the detector at interaction with electrons change the electrical characteristics of the detector, which is reflected in the measured electrical signal. In thermodetectors the absorption of photons leads to an increase of the temperature and temperature characteristics of the detector. Thus, the pyroelectric detector measures the change in internal electron polarization; in bolometers - electrical resistance, etc. The photodetectors have, as a rule, better sensitivity and are more widespread, whereas temperature detectors are used mainly for standard measurements. Photodetectors are divided into three classes: photoemission, semiconductor and superconducting detectors. These types of photodetectors may be used in hybrid devices; photoemission electrons may be recorded when they are exposed to a semiconductor CCD (EBCCD type detectors and ICSD). In photoemission detectors incident photon knocks an electron from the surface of the photocathode, which is accelerated under electric field in vacuum and moves to the anode. The resulting electric current of the detector is proportional to the number of photoelectrons, i.e. the intensity of the incident radiation. The effectiveness of photoemission and spectral energy characteristics of such a detector are defined by the working surface of the photocathode. Advantages of such photodetectors are: high sensitivity and convenience; disadvantages: low quantum efficiency, the spectral dependence of the detector response and the dependence of its efficiency on surface cleanliness. In semiconductor detectors, photons are absorbed within the volume of the semiconductor material, creating a pair of "electron-hole" and the corresponding conductivity. This class of detectors uses an internal photoelectric effect, in which the photon energy must be large enough to overcome the photoelectron band gap to be moved to the conduction band. In photodiodes, Schottky diodes and metal-insulator-semiconductors overcome the potential barrier when the external electric field is applied. External detector photocurrent is proportional to the number of detected photons. Advantages of such photodetectors: a wide operating range, linearity, high quantum efficiency, wide dynamic range, large image matrices; disadvantages - aging effects by UV radiation. Superconducting photon detectors - temperature detectors based on the change in the physical state of matter at increased internal energy of the material due to the absorption of UV photons. The following demands are usually put forward to photodetectors:

- Low sensitivity to visible light (solar-blind). Outside the Earth's atmosphere per one photon in the range of 100-200 nm correspond 104-106 photons in the visible and infrared ranges. This ratio is increased on the surface of the Earth. Therefore, the use of transmissive UV-filters with attenuation of signal on $10^{-4}-10^{-3}$ level is not enough, if the detector has good sensitivity in the visible and/or infrared ranges;

- High detection quantum efficiency (DQE). In optical systems, the UV range, where there are large losses in transmission, this value may differ from the quantum efficiency of the photocathode, or CCD. As a result, special filters and windows are used to block visible light DQE of photoemission detectors decreases by an order from $~ 50 \%$ to $2-5 \%$;

- Wide local dynamic range: a maximum value of the ratio of flux in a given point of the detector to a minimum signal level composed $\sim 3$ units of the noise signal. At the integration over the detector area, the integral dynamic range of the detector is obtained. This option is especially important for sensitive photomultiplier detectors and the development of high-speed position-sensitive detectors;

- Low level of background noise and signal that determines the practical sensitivity of the device and the image contrast.

It should be mentioned that biophotonic emition is measured to be much weaker compared to other types of radiation. Biophotons according to recent studies have a wavelength from $200 \mathrm{~nm}$ to $800 \mathrm{~nm}(0.2-0.8 \mu \mathrm{m})$ (Devaraj \& Inaba, 1997; Kim, 2002.). They are usually being observed in the close UV range (from $300 \mathrm{~nm}$ to $400 \mathrm{~nm}$ ) and visible optic range (from $0.38 \mu \mathrm{m}$ to $0.75 \mu \mathrm{m}$ ) with frequencies ranges from $10^{-19}$ to $10^{-16} \mathrm{~W} / \mathrm{cm}^{2}$ (approx. 1-1000 photons $\mathrm{cm}^{-2}$. $\mathrm{sec}^{-1}$ ) (Inaba, 2000). This light intensity is much weaker than the one that may be 
seen in the perceptually visible and normal bioluminescence, but is detectable above the background of thermal or infrared $(0.74-2.5 \mu \mathrm{m})$ radiation emitted by tissues at their normal temperature. F.A. Popp proposed that this type of light is not radiated in a dispersed way as daylight, but quite coherently maintaining order in the flow oscillations, stability and continuity of the phase difference of the amplitude of the total wave, e.g. it might be both semi-periodic and coherent (Popp et al., 1992). It is known that coherence is an ideal property of waves that enables stationary (i.e. temporally and spatially constant) interference. More generally, coherence describes all properties of the correlation between physical quantities of a single wave, or between several waves or wave packets (Beloussov et al., 2000). This may signify that biophotones in the light's beam vibrate simultaneously, like in a laser beam that can indicate on information characteristics of the signal. The photon emission in its turn is weaker than normal bioluminescence because during that process individual photons are being emanated (Lin, 2006). The "delayed luminescence" is connected with hyperbolic relaxation of biological objects that is a characteristic active response of coherent states. In recent years the evidence has been found that the light has a high degree of coherence because of its photon count statistics, the spectral distribution, unstable decay behavior after exposure to light illumination, and its transparency through optically thick materials. Moreover, DNA is apparently an important source of biophoton emission, since conformational changes of DNA induced with 3,8diamino-5-ethyl-6-phenylphenanthridinium bromide in vivo are clearly reflected by changes of photon emission in cells (Rattemeyer et al., 1981). The physical properties of the emission are described, using the DNA molecule as an exciplex ultraviolet laser system, where a stable state can be reached far from thermal equilibrium at threshold. (Popp et al., 1994) One of us, Ch. Drossinakis, has performed the scrutinized studying of luminescence emission in over 60 scientific institutes, and part of them on the topic of bioluminescence. He used special photoemission detector for detection of strongly expressed biophotone radiation emitted from the human body. These results are shown on Fig. 1 and Fig. 2.

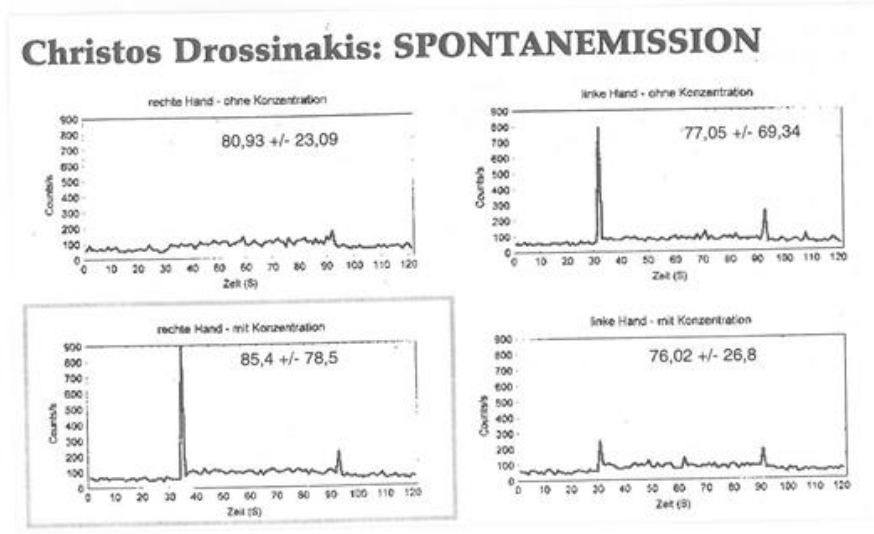

Fig. 1. The results of $\mathrm{Ch}$. Drossinakis on research of spontaneous biophoton emission from the human body 


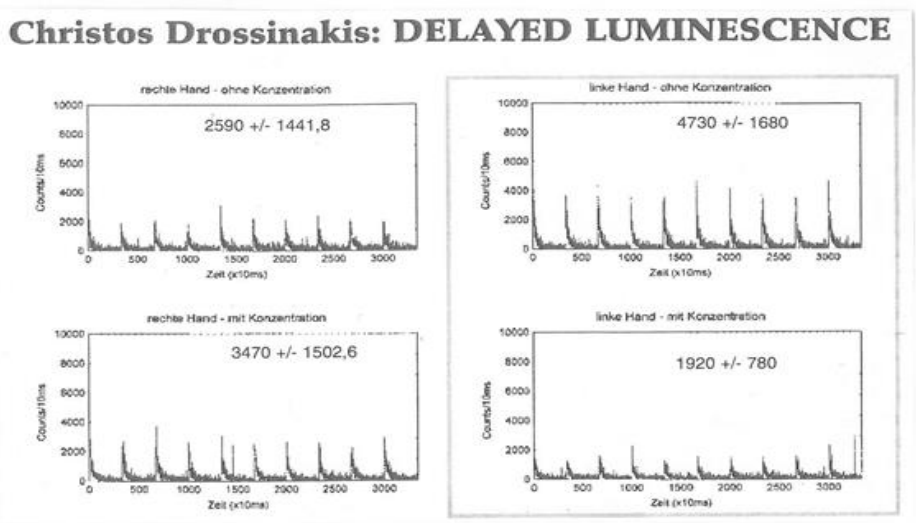

Fig. 2. The results of Ch. Drossinakis on research of delayed luminescence from the human body

It was shown that $1 \mathrm{~cm}^{2}$ of skin generally emits $\sim 85$ photones for $1 \mathrm{~s}$. The intensity of biophoton emission ranges from $10^{-19}$ to $10^{-16} \mathrm{~W} / \mathrm{cm}^{2}$ (approx. $\sim 1-1000$ photons $\mathrm{cm}^{-2} \mathrm{~s}^{-1}$ ) and depends on a number of conditions as intensity of biochemical processes, metabolism, temperature etc. The result of Ch. Drossinakis shows $\sim 900$ biophotons from $1 \mathrm{~cm}^{2}$ of the skin for $1 \mathrm{~s}$. However, there are no reliable proofs that this method has consistent results for medical diagnostics.

\subsection{Color coronal discharge spectral analysis}

Coronal gas discharge effect is indicated by the glow corona electrical discharge (flooding, crown, streamer) on the surface of objects being placed in the alternating electric field of high frequency $(10-150 \mathrm{kHz})$ and electric voltage $(5-30 \mathrm{kV})$ (Kilrian, 1949). In this process in the ionization zone develops the gas corona discharge sliding on dielectric surface, occurring in a nonuniform electric field near the electrode with a small radius of curvature. In the thin air layer with thickness of $\sim 10-100 \mu \mathrm{m}$ between the studied object and the electrode are developed the following processes:

- Excitation, polarization and ionization by electric field of high frequency the main components of air - the molecules of nitrogen $\left(78 \% \mathrm{~N}_{2}\right)$, oxygen $\left(21 \% \mathrm{O}_{2}\right)$ and carbon dioxide $\left(0.046 \% \mathrm{CO}_{2}\right)$. As a result of this is formed an ionized gas, i.e. gas with separated electrons having negative charges, creating a conductive medium as plasma;

- Formation of a weak electric current in the form of free electrons separated from molecules of $\mathrm{N}_{2}, \mathrm{O}_{2}$ and $\mathrm{CO}_{2}$, which generate gas discharge between the studied object and the electrode. The form of gas discharge glowing, its density and surface brightness distribution is determined mainly by electromagnetic properties of the object;

- The transition of electrons from lower to higher energy levels and back again, during which there appears a discrete quantum of light radiation in the form of photon radiation. The transition energy of electrons depends on the external electric field and the electronic state of the studied object. Therefore, in different areas surrounding the electric field, the electrons receive different energy impulses, i.e. "skipping" at different energy levels that result in emission of photons with different wavelengths (frequencies) and the energy, coloring the contour of the glow in various spectral colors.

Processes outlined above form the total gas electric effect (Ignatov \& Mosin, 2012), which allows the studying of the electrical properties of the object at its interaction with an external electromagnetic field (Ignatov \& Mosin, 2013a; Ignatov \& Mosin, 2013b). It was shown that the electrical conductivity of the object has almost no effect on the formation of the electric images, which mostly depends on the dielectric constant (Pehek et al., 1976).

There is a relationship (1) of the electric discharge per unit area of the recording medium on the following parameters:

$\sigma=\left[\alpha-U_{p}\left(d_{2}+\delta\right) / d_{2}\right] \varepsilon_{0}\left(d_{2}+\delta\right) / \delta d_{2}, \quad(1) \quad$ where: $\delta=d_{1} / \varepsilon_{1}+d_{3} / \varepsilon_{3}$ 
$\alpha$-slope rate of electrical pulse;

$\mathrm{T}$ - duration of the electrical pulse;

$\mathrm{U}_{\mathrm{p}}-$ breakdown voltage of the air layer between the object and the recording medium;

$\mathrm{d}_{1}$ - the width of the object;

$\mathrm{d}_{2}$ - width of the zone of influence of the electromagnetic field;

$\mathrm{d}_{3} \quad$ - width of the recording medium;

$\varepsilon_{0}-$ dielectric permittivity of the air $\left(\varepsilon_{0}=1.00057 \mathrm{~F} / \mathrm{m}\right)$;

$\varepsilon_{1}$ - dielectric permittivity of the studied object;

$\varepsilon_{3}-$ dielectric permittivity of the medium.

To calculate the breakdown voltage of the air layer, this formula is used:

$U_{p}=312+6,2 d_{2}$

As a result of mathematical transformations is obtained a quadratic equation describing the width of the air layer:

$6,2 d_{2}^{2}-(\alpha T-6,2 \delta-312) d_{2}+312 \delta=0$

This equation has two solutions:

$d_{2}=[\alpha T-6,2 \delta-312] \pm\left[(\alpha T-6,2 \delta-312)^{2}-7738 \delta\right)^{1 / 2}$

The above equations allow calculating maximum and minimum width of the air layer for the occurrence of elecric discharge under which is being formed the electrical image of the studied object. Gas discharge characteristics for various biological objects vary in character and light intensity, size of contour glow and color spectrum and depend both on its own electromagnetic radiation and the dielectric constant of the object. The intensity depends on the electric voltage applied on the electrode. Studies have shown that the contours of gas discharge glow at $12 \mathrm{kHz}$ and $15 \mathrm{kHz}$ and are homogeneous in their structure. The contour at $\mathrm{kHz}$ is $55 \%$ of the contour at $15 \mathrm{kHz}$ and at $24 \mathrm{kHz}$ - only $15 \%$ of the contour at $15 \mathrm{kHz}$ that is important for further analysis and identification of images. The incidence of bioelectrical activity of the body reduces the intensity of gas discharge glow. Pathology in the organism and surrounding tissues also alter the bioelectric activity and the shape and color of gas discharge glow, which is determined mainly by energy of photon emission at the transition of electrons from higher energy levels to the lower ones when being excited by the external electric field. Thus, for red colour of the electromagnetic spectrum this energy compiles $1.82 \mathrm{eV}$, for orange color $-2.05 \mathrm{eV}$, yellow $2.14 \mathrm{eV}$, blue-green (cyan) $-2.43 \mathrm{eV}$, blue $-2.64 \mathrm{eV}$, and violet $-3.03 \mathrm{eV}$. The reliable result norm is at $\mathrm{E} \geq 2.53$ $\mathrm{eV}$. The spectral range of the photon emission for different colors is within 380-495 nm and 570-750 $\mathrm{nm} \pm 5 \mathrm{~nm}$. The photons, corresponding to the emission with green color in the visible electromagnetic spectrum, are not being detected under those experimental conditions. Thus, the more predominant in the color spectrum yellow, orange, blue-green, blue and violet colors, the more pronounced the gas discharge glow and bioelectric properties of the object. According to the data obtained, the incidence of bioelectrical activity of the body reduces the intensity of gas discharge glow. Studies carried out by I. Ignatov on 2125 people show that the overall drop in the bioelectric activity of the body, as well as pathology in the organism alter the bioelectric activity and reduce the apparent size of the gas disharge glow. This dependence is observed for many disorders, although there are not statistical reliable results that this method can be applied in medical diagnostics. Fig. 3 shows the results on bioelectrical discharge images of thumbs of various people detected on color photofilm under coronal gas discharge conditions. The research area was from part of the thumb contacted with transparent electrode. The norm of energy of photon emission compiles $2.54 \mathrm{eV}$. If the value is over $2.54 \mathrm{eV}$, this is an indicator of normal bioelectrical status. Some people with high energy status possess the values of photon emission over $2.90 \mathrm{eV}$. The high values of this parameter are possible with the practicing of yoga, sport, etc. The emission less than $2.53 \mathrm{eV}$ is characteristic for people with low bioelectrical status. These results are interesting from scientific point of view, because they may provide prospects for further using of this method for 
biophysical studies.
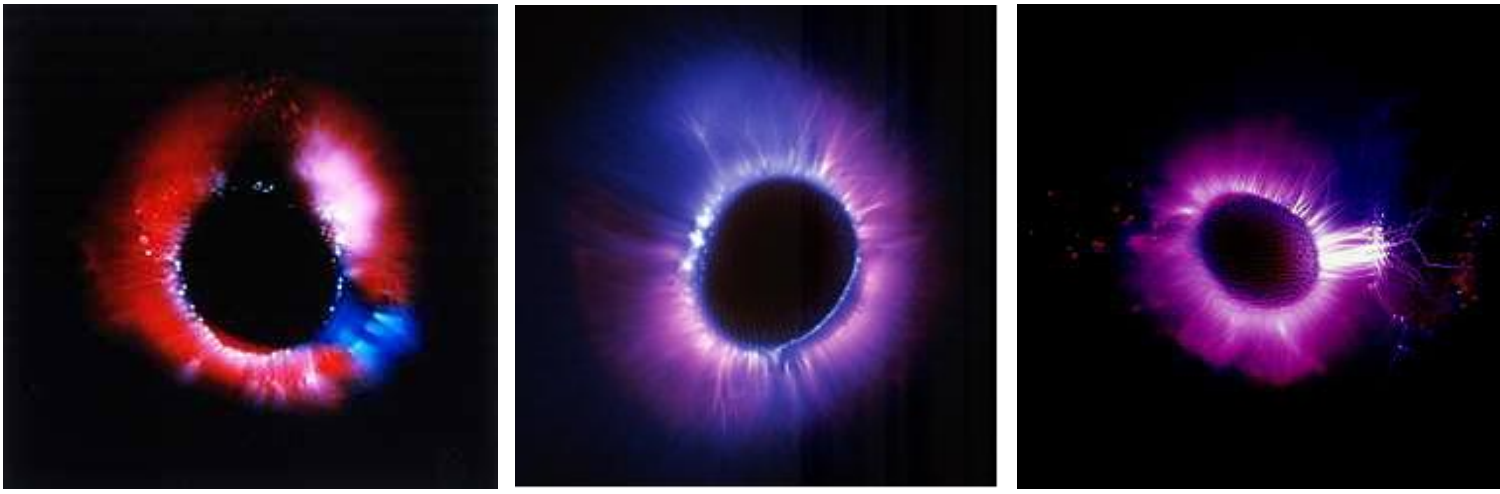

a). Normal bioelectrical discharge b). Bioelectrical discharge image image of a person $(1.94 \mathrm{eV}) \quad$ of Valentina Tomova $(2.90 \mathrm{eV})$

c). Bioelectrical discharge image of an of Lara Weigmann $(3.00 \mathrm{eV})$
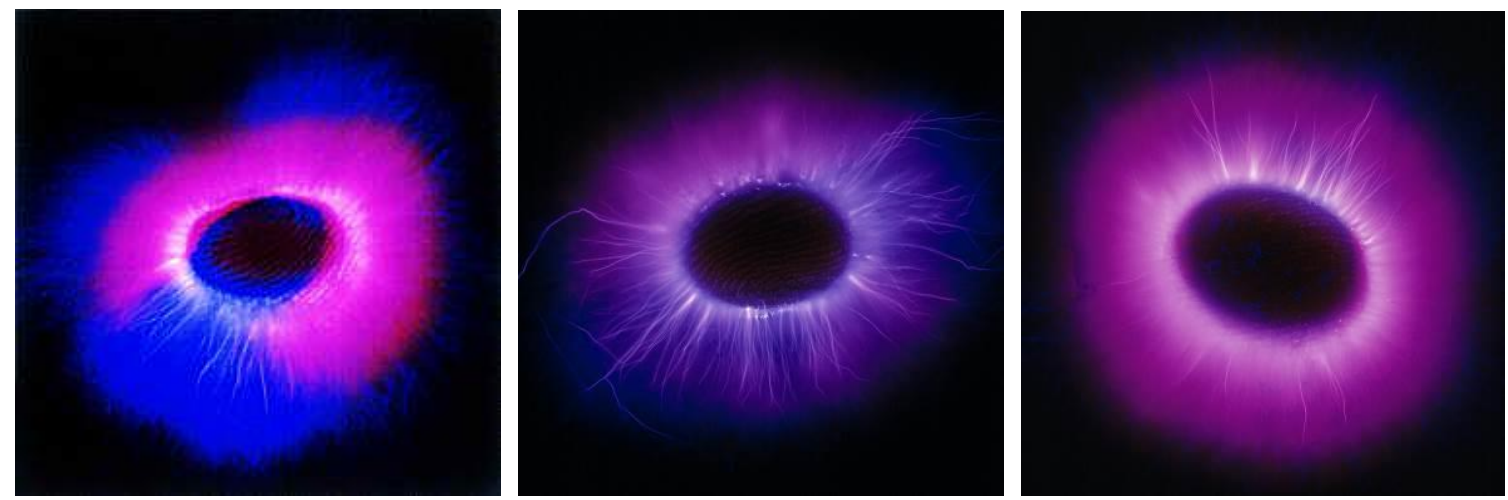

d).Bioelectrical discharge image

e).Bioelectrical discharge image $f$ ). Bioelectrical image of Lieselotte Eder $(2.91 \mathrm{eV})$ of Marlen Vinchere Robayo (2.95eV) Dr. Pascal Boesinger (3.03 eV)

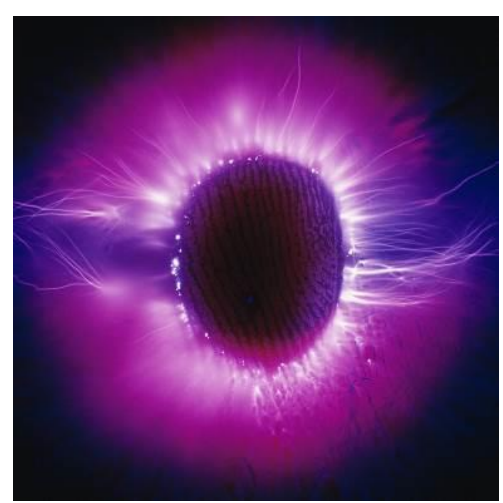

.$g$ ). Bioelectrical discharge image Dipl. Eng. Enrico Bauer (3.00eV)

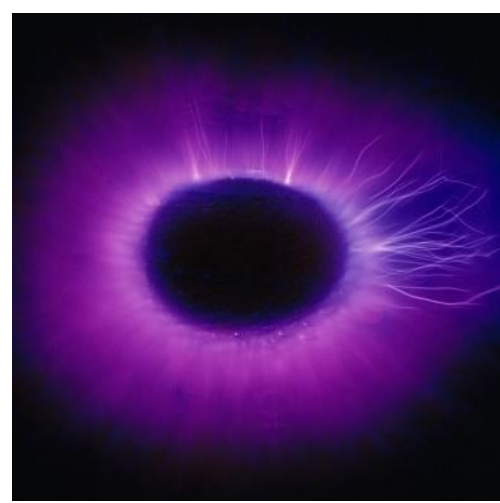

h). Bioelectrical discharge image of Karin Mueller (2.98 eV)

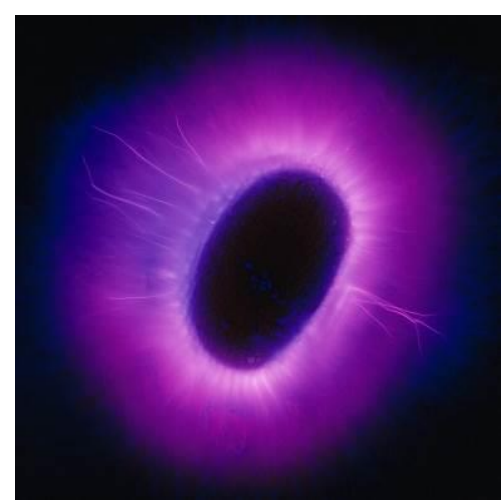

i). Bioelectrical discharge image of of of Gerda Salzmann (3.01 eV)

Fig. 3. Bioelectrical discharge images of thumbs of people relative to the bioelectrical discharge image of a normal person (I. Ignatov)

\subsection{NES and DNES analysis of water}

Water seems to be a good model system for studying the interaction with electromagnetic fields and structural 
research. Recent data indicated that water is a complex associated non-equilibrium liquid consisting of associative groups (clusters) containing from 3 to 50 individual $\mathrm{H}_{2} \mathrm{O}$ molecules (Keutsch \& Saykally, 2011). These associates can be described as unstable groups (dimers, trimers, tetramers, pentamers, hexamers etc.) in which individual $\mathrm{H}_{2} \mathrm{O}$ molecules are linked by van der Waals forces, dipole-dipole and other charge-transfer interactions, including hydrogen bonding (Ignatov \& Mosin, 2013c). At room temperature, the degree of association of $\mathrm{H}_{2} \mathrm{O}$ molecules may vary from 2 to 21 . The measurements were performed with NES and DNES methods. It was established experimentally that in the process of evaporation of water drops, the wetting angle $\theta$ decreases discreetly to 0 , and the diameter of water drop basis is only slightly altered, that is a new physical effect (Antonov \& Yuskesselieva, 1983). Based on this effect, by means of measurement of the wetting angle within equal intervals of time is determined the function of distribution of $\mathrm{H}_{2} \mathrm{O}$ molecules according to the value of $f(\theta)$. The distribution function is denoted as the energy spectrum of the water state. A theoretical research established the dependence between the surface tension of water and the energy of hydrogen bonds among individual $\mathrm{H}_{2} \mathrm{O}$-molecules (Antonov, 1995). The hydrogen bonding results from interaction between electron-deficient $\mathrm{H}$-atom of one $\mathrm{H}_{2} \mathrm{O}$ molecule (hydrogen donor) and unshared electron pair of an electronegative $\mathrm{O}$-atom (hydrogen acceptor) on the neighboring $\mathrm{H}_{2} \mathrm{O}$ molecule; the structure of hydrogen bonding may be defined as $\mathrm{O} \cdots \cdot \mathrm{H}^{\mathrm{n}+}-\mathrm{O}^{\mathrm{n}-}$. For calculation of the function $\mathrm{f}(\mathrm{E})$ represented the energy spectrum of water, the experimental dependence between the wetting angle $(\theta)$ and the energy of hydrogen bonds $(E)$ is established:

$\left.f(E)=b \times f(\theta) / 1-(1+b \times E)^{2}\right)^{1 / 2},(5)$

where $\mathrm{b}=14.33 \mathrm{eV}^{-1}$ (6)

The relation between the wetting angle $(\theta)$ and the energy (E) of the hydrogen bonds between $\mathrm{H}_{2} \mathrm{O}$ molecules is calculated by the formula:

$\theta=\operatorname{arcos}(-1-14.33 \mathrm{E})(7)$

The energy spectrum of water is characterized by a non-equilibrium process of water droplets evaporation, therefore, the term non-equilibrium spectrum (NES) of water is used. The energy of hydrogen bonds measured by NES is determined as $\overline{\mathrm{E}}=-0,1067 \pm 0,0011 \mathrm{eV}$.

The difference $\Delta f(E)=f($ samples of water $)-f($ control sample of water $)$

- is called the "differential non-equilibrium energy spectrum of water" (DNES).

Thus, DNES spectrum is an indicator of structural changes of water as a result of various external factors. The cumulative effect of these factors is not the same for the control sample of water and the water sample being under the influence of this factor. Fig. 4 shows NES-spectrum of deionized water that was used as a model system for studying the interaction of electromagnetic field with water. On the X-axis are given three scales. The energies of hydrogen bonds among $\mathrm{H}_{2} \mathrm{O}$ molecules are calculated in eV. On the Y-axis is shown the energy distribution function $\mathrm{f}(\mathrm{E})$ of $\mathrm{H}_{2} \mathrm{O}$ molecules measured in $\mathrm{eV}^{-1}$. It was shown that the window of transparency of the earth atmosphere for the electromagnetic radiation in the middle IR-range almost covers NES-spectrum of water. Arrows $\mathrm{A}$ and $\mathrm{B}$ designate the energy of hydrogen bonds among $\mathrm{H}_{2} \mathrm{O}$ molecules. Arrow $\mathrm{C}$ designates the energy at which the human body behaves itself as absolute black body (ABB) at optimum temperature $36.6{ }^{0} \mathrm{C}$ and adsorbs the thermal radiation. A horizontal arrow designates the window of transparency of the earth atmosphere for the electromagnetic radiation in the middle IR-range. 


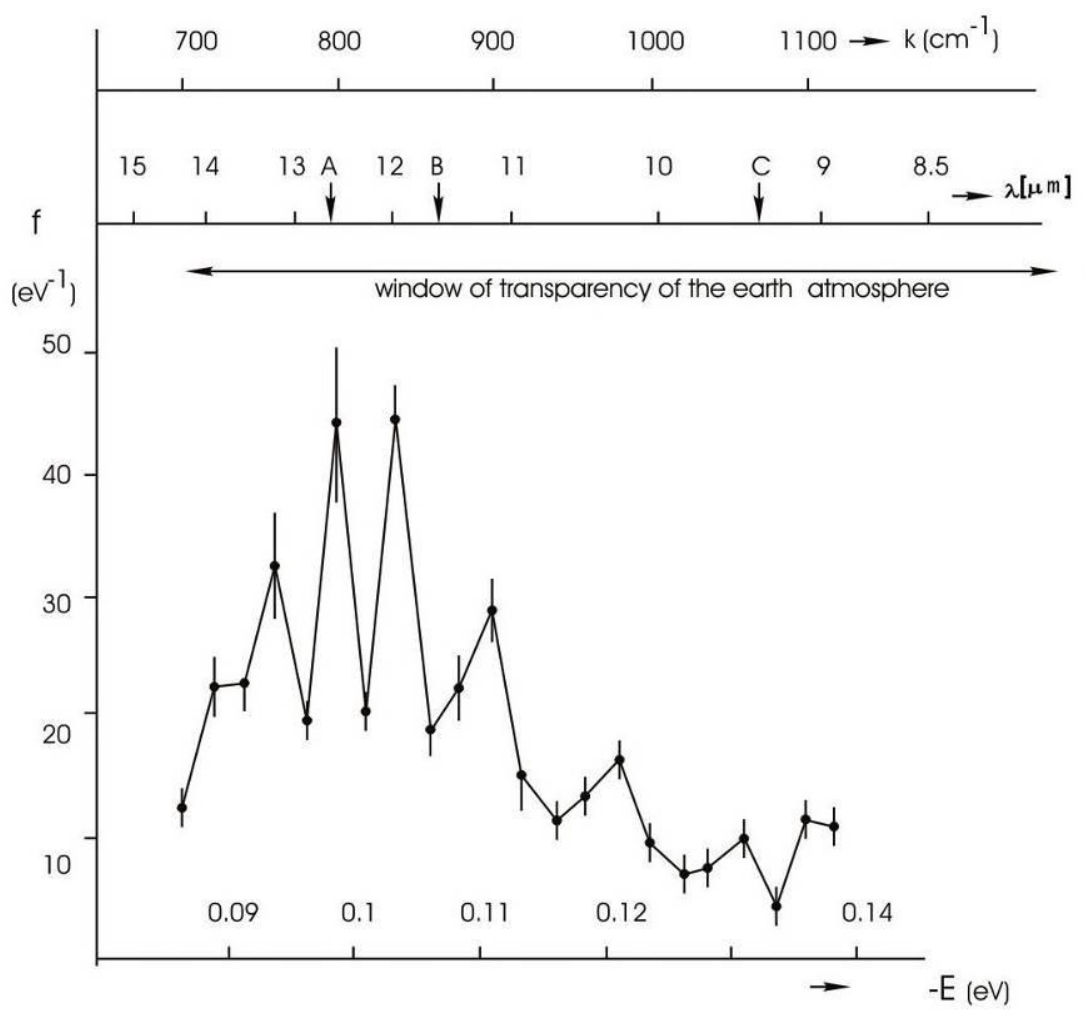

Fig. 4. Non-equilibrium energy spectrum (NES) of water as a result of measurement for 1 year: $\lambda-$ wavelength, $k$ - wave number.

Another important physical parameter was calculated with NES and DNES methods - the average energy $\left(\Delta \mathrm{E}_{\mathrm{H} \ldots \mathrm{O}}\right)$ of $\mathrm{H} \ldots \mathrm{O}$-bonds between $\mathrm{H}_{2} \mathrm{O}$ compiled $-0.1067 \pm 0.0011 \mathrm{eV}$. The most remarkable peculiarity of $\mathrm{H}$...O-bond consists in its relatively low strength; it is 5-10 times weaker than chemical covalent bond. In respect of energy hydrogen bond has an intermediate position between covalent bonds and intermolecular van der Waals forces, based on dipole-dipole interactions, holding the neutral molecules together in gasses or liquefied or solidified gasses. Hydrogen bonding produces interatomic distances shorter than the sum of van der Waals radii, and usually involves a limited number of interaction partners. These characteristics become more substantial when acceptors bind $\mathrm{H}$ atoms from more electronegative donors. Hydrogen bonds hold $\mathrm{H}_{2} \mathrm{O}$ molecules on $15 \%$ closer than if water was a simple liquid with van der Waals interactions. The hydrogen bond energy compiles $5-10 \mathrm{kcal} / \mathrm{mole}$, while the energy of covalent $\mathrm{O}-\mathrm{H}$-bonds in $\mathrm{H}_{2} \mathrm{O}$ molecule $-109 \mathrm{kcal} / \mathrm{mole}$. With fluctuations of water temperature the average energy of hydrogen $\mathrm{H}$...O-bonds in $\mathrm{H}_{2} \mathrm{O}$ molecule associates changes. That is why hydrogen bonds in liquid state are relatively weak and unstable: it is thought that they can easily form and disappear as the result of temperature fluctuations. The next conclusion that can be drawn from our research is that there is the distribution of energies among individual $\mathrm{H}_{2} \mathrm{O}$ molecules. Further we performed two types of temperature-dependent experiments on heat exchange from the surface of the human body by DNES-method. In the first experiment we studied heat exchange when the temperature of the human body was higher than the temperature of the surrounding environment (curve 1a and $1 \mathrm{~b}$ on Fig. 5). In the second experiment there was heat exchange when the temperature of the human body was lower than that of the surrounding environment (curve $2 a$ and $2 b$ on Fig. 5). In both experiments a local maximum was detected at 9.7 $\mu \mathrm{m}$ on curve 1 and curve 2 (Fig. 5). This local maximum corresponds to the maximal level of heat emission from the surface of the human body and lays within the "transparency window" of the Earth atmosphere to electromagnetic radiation in the mid IR-range of the electromagnetic spectrum. In this range, the electromagnetic radiation emitted by the Earth in the surrounding space is being absorbed by the Earth atmosphere. There is a statistical difference between the results of heat emission from the surface of the human body to the surrounding environment and back to the human body according to the $t$-criterion of Student at $\mathrm{p}<0.01$. The local maximum 
on curve $1 \mathrm{a}$ is detected at $7.3 \mathrm{eV}^{-1}$, while the local maximum on curve $2 \mathrm{a}-$ at $2.4 \mathrm{eV}^{-1}$ (Fig. 5).

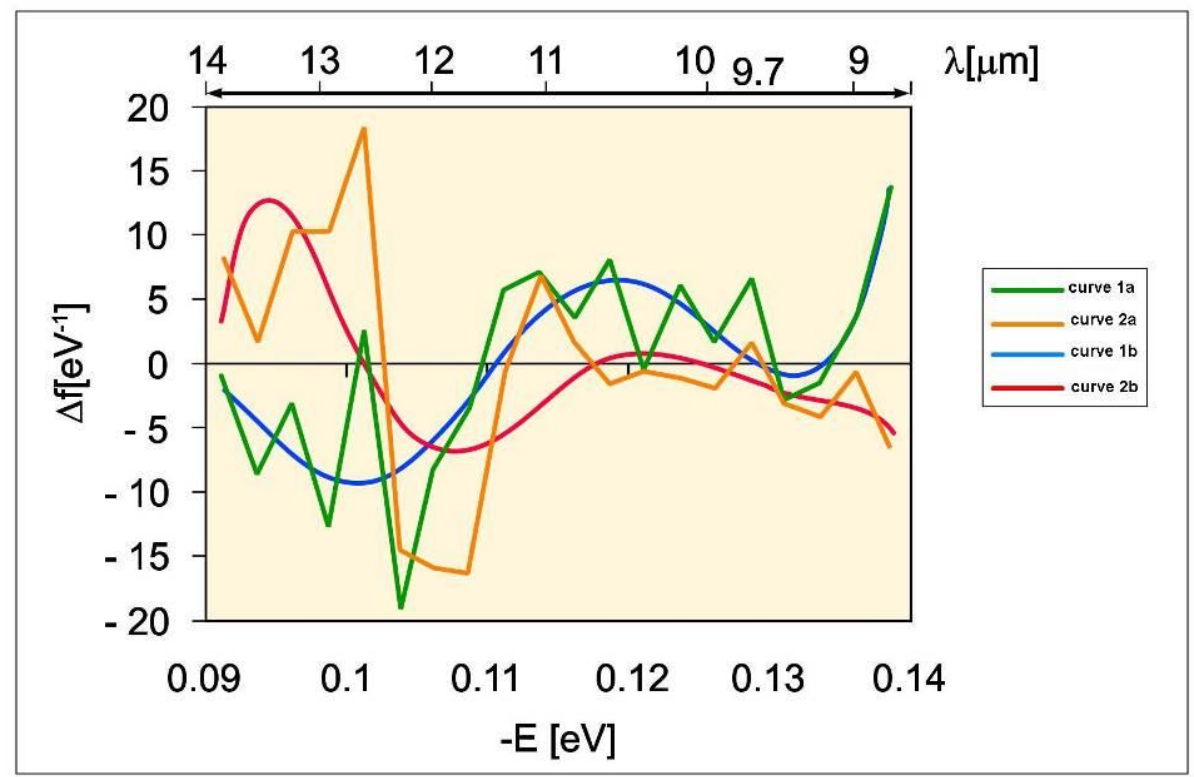

Fig. 5. Differential non-equilibrium energy spectrum (DNES) reflecting the heat exchange of the human body with the surrounding environment.

\subsection{Infrared thermography (IRT)}

The human body as a biological body has an average temperature in the range from 36.6 to $36.8{ }^{0} \mathrm{C}$, The main part of this radiation predominantly falls on human skin with a long wavelength range from 4 to $50 \mu \mathrm{m}$. Maximum of spectral density covers the range approx. $\sim 10 \mu \mathrm{m}$ i.e. the middle wavelength IR range. The physical essence of the thermal radiation consists in the presence of charged particles (electrons and ions), which are in random motion and have the properties of electrical or magnetic polarity. Infrared radiation is emitted or absorbed by excited atoms or ions when they change their rotational-vibrational movements. Electromagnetic waves propagate throughout the body and reach the surface, passing through the skin and partly emitted into the environment. The intensity of these processes is proportional to the body temperature. The wavelength of infrared radiation emitted by the body depends on the heating temperature: the higher the temperature, the shorter is the wavelength and therefore the higher the emission intensity. Studies have shown that in the long wavelength infrared region $(8-14 \mu \mathrm{m})$ the human skin radiates as a black body, regardless of age, degree of pigmentation and other features. Therefore, the emissivity of the human skin can be considered equal to 1 absolute unit. In practice, it is proved that the difference between the emission characteristics of the human skin and blackbody still exist, however, it is small and depends essentially on the influence of the surrounding background. The limit of effective temperature measurement is equal to the thickness of the emitting layer (skin layer) and is defined as the distance at which electromagnetic waves propagate from the object's surface before the layer in which the intensity decreases in $\sim 2.5$ times. Under equal conditions, the greater the wavelength, the greater the depth, which can detect the temperature perturbations. The maximum intensity of thermal radiation at normal ambient temperature is located in the infrared rage of the spectrum (wavelength $\sim 10 \mu \mathrm{m}$ at $\mathrm{t}=36.6{ }^{0} \mathrm{C}$ ). The threshold of skin sensitivity according to Yu.V. Gulyaev and E.E. Godik compiles $\sim 10^{-14} \mathrm{~W} / \mathrm{cm}^{2}$ (Gulyaev \& Godik, 1990). This led to the feasibility of establishing IR thermal imaging (thermography) for the study of the temperature anomalies. However, the measurement of the thermal radiation of the human body in the IR range gives the true temperature for only the top layer of skin with thickness of $\sim 1 \mathrm{~mm}$; after that the thermal radiation is reflected back into the environment. The temperature of the underlying tissues and organs can be judged indirectly when the temperature changes are "projected" on the skin. Infrared thermography is a scientific method for registering the thermogram - infrared image showing the distribution pattern of infrared waves emitted from the objects (Ring \& Hughes, 1986). Thermographic cameras detect radiation in the infrared range of the electromagnetic spectrum (approx. $\sim 0.9-14 \mu \mathrm{m}$ ), and on its basis are obtained thermographic images (thermograms) allowing to determine the locations of patterns having different temperatures. Thermograms 
therefore are actually visual displays of the amount of infrared energy emitted, transmitted, and reflected from the surface of the object. Since infrared radiation is emitted by all objects with the temperature according to Planck's formula for black body radiation, thermography allows to "see" the environment with or without visible illumination. The intensity of the thermal radiation of the body increases with the temperature, therefore thermography allows to see the temperature distribution on the surface of the body. As a result, warm objects are seen better on the cooler environment background; mammals and warm-blooded animals are better visible on the environment. That is why thermography may find many diagnostic applications and is often being used for breast diagnostics, tumour detection etc. Most thermographic cameras use CCD and CMOS image sensors having most of their spectral sensitivity in the visible light wavelength range. The most commonly used is a matrix of indium antimonide ( $\mathrm{InSb}$ ), gallium arsenide (GaAs), mercury telluride ( $\mathrm{HgTe})$, indium (In) and cadmium (Cd). The latest technology allows the use of the inexpensive uncooled microbolometer sensors. Their resolution is varied from $160 \times 120$ or $320 \times 240$ up to $768 \times 1024$ pixels in the most advanced camera models. Often the thermogram reveals temperature variations so clearly that a photograph is not necessary for further analysis. Usually a block of the focal planes of thermo images can detect radiation in the medium (3 to $5 \mu \mathrm{m})$ and long ( 8 to $15 \mu \mathrm{m}$ ) infrared wave band, designated as MWIR and LWIR corresponding to two infrared windows with high coeficient of transmittance. Improperly selected temperature range on the surface of the objects, indicates a potential problem.

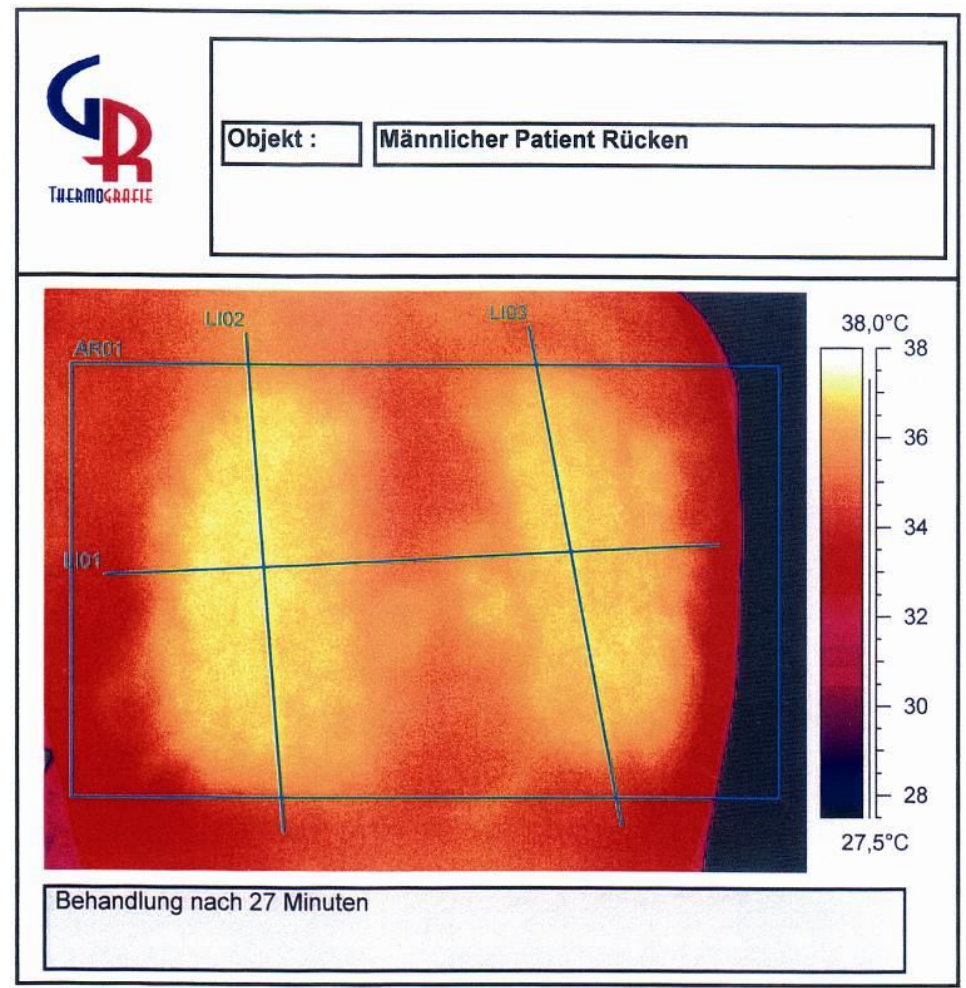

Fig. 6. Thermovisual snapshot of the result of bioinfluence of Ch. Drossinakis on skin section of the back of a person suffering from radiculitis after $27 \mathrm{~min}$

It should be noted that the intensity of the thermal radiation of the human body in the microwave (MW) range is much smaller in magnitude than in the infrared part of the spectrum (Sisodia, 2007). In particular at a wavelength of $17 \mathrm{~cm}$ the intensity is less in $\sim 10$ times, so the heat reception signals in this range of the spectrum require equipment with higher sensitivity. However, the advantage of this method is that the measurement range and the depth of radiation penetration is much greater, therefore it is possible to obtain data on the temperature parameters of the internal organs and structures of the human body, but the resolution is significantly reduced, therefore it is impossible to obtain reliable thermal image of the study area. Infrared thermography registered a thermal infrared radiation emitted by the capillary network of the skin, which is used in medicine for thermovisual diagnostics. The closer an ailing organ is to the skin, the more accurate the diagnosis based on a thermal signal. Today it seems to be an established fact that some people possess the ability to increase the 
temperature of the treated area of the human body. Figure 6 shows the thermovisual result of the temperature difference between the initial $\left(\mathrm{t}=35.6{ }^{\circ} \mathrm{C}\right)$ and final skin section temperature $\left(\mathrm{t}=37.3{ }^{\circ} \mathrm{C}\right)$ of the person before and after the treatment of Drossinakis. It was calculated that the temperature of the skin part was increased after the treatment on $1.7{ }^{0} \mathrm{C}$. In this connection there should be noted two important empirical thermography results obtained by M. Marinov (Marinov \& Ignatov, 2008), which allow the medical diagnostics of various human organs and monitoring of their condition and malfunction by this method. Fig. $7 a$ shows the thermography snapshot of a patient having a benign tumour growth in the mammary gland, which has a higher temperature than the surrounding tissues' lower temperature with $0.54{ }^{\circ} \mathrm{C}$. Fig. $7 b$ shows a patient having hyperfunction of thyroid gland, which has higher temperature than the surrounding tissues' lower temperature at $0.76{ }^{0} \mathrm{C}$. The middle value on the scale is $36.6{ }^{\circ} \mathrm{C}$. In the left side of the scale there are temperatures less than $36.6{ }^{\circ} \mathrm{C}$. In the right side of the scale there are temperatures more than $36.6{ }^{0} \mathrm{C}$.

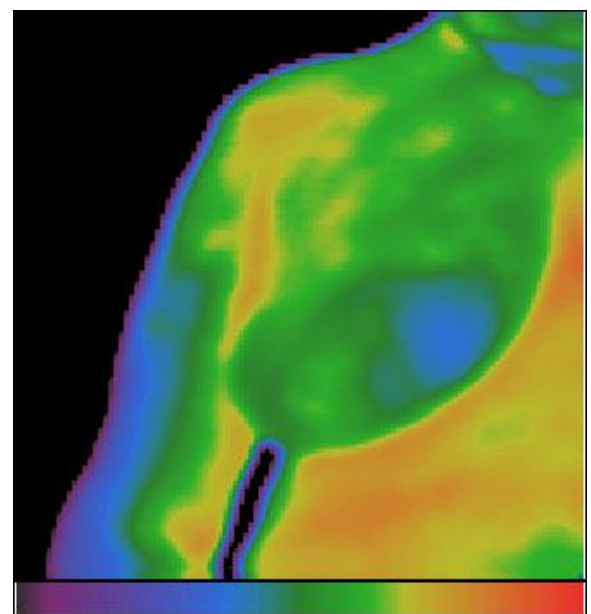

a)

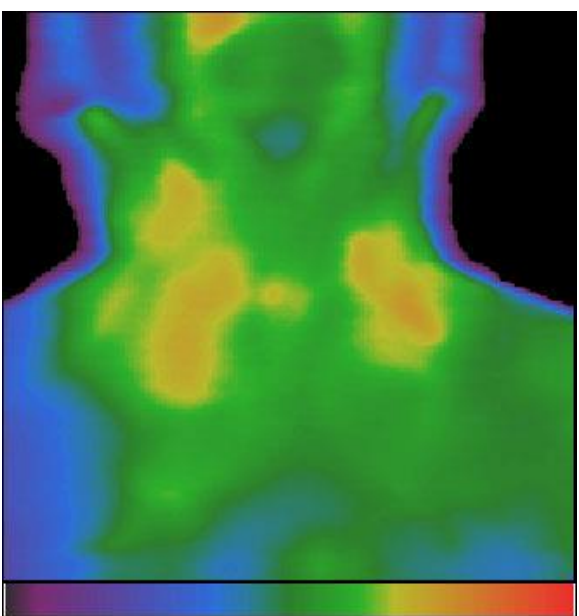

b)

Fig. 7. Thermovisual snapshots of the human body (Marinov, 2008): $a$ ) - patient having a benign tumor growth in the mammary gland; $b$ ) - patient having hyperfunction of thyroid gland

\section{Conclusions}

Within the frames of this research many types of NIR radiation (electromagnetic waves, infrared radiation, thermo radiation, bioluminescence) emitted from the human body were studied and carefully scrutinized. The approaches and methods for detecting various types of radiation employed in this research as magnetography, infrared thermography, chemiluminescence and coronal gas discharge spectral analysis can find further application in many branches of applied science and medical diagnostics, while other methods as NES and DNES may be applied for studying the interaction of electromagnetic fields with water and structural studies.

\section{Acknowledgements}

The authors wish to thank Alexander Popp for kindly providing the scientific correspondence and letters of his father, Prof. Fritz-Albert Popp. The authors also wish to thank Dipl. Eng. Charlie Stoyanov, co-author of Ignatov for the structuring of the device for color coronal gas discharge. The authors are thankful to Vassil Marinov from $22^{\text {nd }}$ Clinical and Diagnostical Medical Center, Sofia, Bulgaria for clinical data with thermo camera. The authors are thankful to assistant Lara Weigmann and the students of Christos Drossinakis - Pascal Boesinger, Marlen Vinchere Robayo, Enrico Bauer, Gerda Salzmann, Karin Mueller.

\section{References}

Adey, W.R. (1981) Tissue interaction with non-ionizing electromagnetic fields. Physiol. Rev., 61: 435-514. Anderson, L.E. (1993) Biological effect of extremely low frequency electromagnetic fields: in vivo studies. Am. Ind. Hig. Assoc. J., 54: 186-196.

Anosov V.N. \& Trukhan E.M. (2003) A new approach to the problem of weak magnetic fields: An effect on living objects. Doklady Biochemistry and Biophysics, 392(1-6): 274-278.

Antonov, A. \& Yuskesselieva, L. (1985) Selective high frequency discharge (Kirlian effect). Acta Hydrophysica, 
Berlin, 5: 29.

Antonov, A. (1995) Research of the non-equilibrium processes in the area in allocated systems. Dissertation thesis "Doctor of physical sciences", Blagoevgrad, Sofia.

Barnes, F.S. \& Greenebaum, B. (eds.) (2006) CRC Handbook on biological effects of electromagnetic fields. 3d Edition, Boca Raton: CRC Press, November 2006, Vol. 2, 960 p.

Bars, Le. \& Andre, G. (1976) Biological effects of electric fields on rats and rabbits. Red. Gen. Elect. (special issue), July 1976: 91-97.

Beloussov, L.V., Opitz, J.M. \& Gilbert, SF (1997) Life of Alexander G. Gurwitsch and his relevant contribution to the theory of morphogenetic fields. The International journal of developmental biology, 41(6): 7-771.

Beloussov, L., Popp, F.A., Voeikov, V., van Wijk, R. (eds) (2000) Biophotonics and Coherent Systems. Moscow, Moscow University Press, 133 p.

Boveris, A., Cadenas, E., Reiter, R., Filipkowski, M., Nakase, Y. \& Chance, B. (1980) Organ chemiluminescence: Noninvasive assay for oxidative radical reactions. Proc. Natl. Acad. Sci. USA, 77: 347-351.

Chang, J.J., Fisch, J. \& Popp, F.A. (eds) (1998) Biophotons. Dordrecht, Kluwer Academic Publishers, 417 p.

Choi, C., Woo, W.M., Lee, M.B. at al. (2002) Biophoton emission from the hands. J. Korean Physical. Soc., 41:275-278.

Cleary, S.F. (1993) A review of in vitro studies: low-frequency electromagnetic fields. J. Am. Ind. Hyg. Assoc, 54(4): 178-185.

Cohen, D. (1968) Magnetoencephlalography: evidence of magnetic fields produced bi alpha-rhythm currents. Science, 161(3843): 784-786.

Cohen, S. \& Popp, F.A. (1997) Biophoton emission of the human body. Journal of Photochemistry and Photobiology B: Biology, 40(2): 187-189.

Devaraj, B., Usa, M. \& Inaba, H. (1997) Biophotons: ultraweak light emission from living systems. Curr. Opin. Solid State Mater Sci., 2:188.

Dobrin, R., Kirsch, C., Kirsch, S. et al. (1979) Experimental measurements of the human energy field. In S. Krippner (ed.), Psychoenergetic Systems: The Interface of Consciousness, Energy and Matter. New York, Gordon \& Breach, 230 p.

Edwards, R., Ibison, M.C., Jessel-Kenyon, J. \& Taylor, R.B. (1989) Light emission from the human body. Complement Med. Res., 3:16.

Esterbauer, H., Zollner, H. \& Schaur, R. J. (1990) Aldehydes formed by lipid peroxidation: mechanisms of formation, occurrence, and determination. In Membrane Lipid Oxidation. Boca Raton, CRC Press, 283 p.

Frey, A.H. (1993) Electromagnetic field interactions with biological systems. FASEB Journal, 7(2): $272-281$.

Gerardi, G., De Ninno A., Prosdocimi, M. et al. (2008) Effects of electromagnetic fields of low frequency and low intensity on rat metabolism. Biomagnetic Research and Technology, 6: 3.

Gubkin, A.N. (1978) Electrets. Moscow, Nauka. 192 p.

Gross, B. (1964) Charge storage in solid dielectrics; a bibliographical review on the electret and related effects. New York, Elsevier Pub. Co., 230 p.

Goodman, R., Greenbaum, B. \& Marron, M.T. (1995) Effects of electromagnetic fields on molecules and cells. Int. Rev. Cytol., 158: 279-338.

Gulyaev, Yu.V. \& Godik, E.E. (1984) On the possibilities of the functional diagnostics of the biological subjects via their temporal dynamics of the infrared images. USSR Academy Nauk Proceedings/Biophysics, 277: 1486-1491.

Gulyaev, Yu.V. \& Godik, E.E. (1990) Human and animal physical fields. Scientific American, 5: 74-83.

Gulyaev, Yu.V. \& Godik, E.E. (1991) Functional Imaging of the Human Body. IEEE Engineering in Medicine and Biology, 10: 21-29.

Gurwitsch, A.G. (1959) Die mitogenetische strahlung, ihre physikalische-chemischen grundlagen und ihre anwendung in biologie und medizin, Jena, Germany, Veb G. Fisher.

Gurwitsch, A.G. (1988) A historical review of the problem of mitogenetic radiation. Experientia, 44: 545-550.

Halliwell, B. \& Gutteridge, J.M.C. (1989) Free Radicals in Biology and Medicine (2nd ed.), Oxford, Clarendon Press.

Hastings, J.W. (1983). Biological diversity, chemical mechanisms, and the evolutionary origins of bioluminescent systems. J. Mol. Evol., 19 (5): 309-321.

Holzel, R. \& Lamprecht, I. (1994) Wirkungen elektromagnetischer Felder auf biologische Systeme. Nachrichtentech Elektron, 44(2): 28-32.

Inaba, H. (2000) Measurement of biophotons from human body. J. Int. Soc. Life Inf. Sci., 18:448.

Ignatov, I., Antonov, A. \& Galabova, T. (1998) Medical Biophysics - Biophysical Fields of Man. Gea Libris, 
Sofia: $1-71$

Ignatov, I., Antonov, A. \& Galabova, T. (2002) Scientific Research Studies with Christos Drossinakis (October 2001 - October 2002), Int. Conference "Man and Nature”, SRCMB, Sofia.

Ignatov, I. (2005) Energy Biomedicine, Gea-Libris, Sofia, 1-88.

Ignatov, I. \& Mosin, O.V. (2012) Kirlian effect in biomedical diagnostics and study of bioenergetical properties of biological objects and water. Biomedical Radio electronics, Biomedical Technologies and Radio Electronics, 12: 13-21 [in Russian].

Ignatov, I. \& Mosin, O.V. (2013a) Method for Color coronal (Kirlian) spectral analysis. Biomedical Radio electronics, Biomedical Technologies and Radio Electronics, 1: 38-47 [in Russian].

Ignatov, I. \& Mosin O.V. (2013b) Color crown spectral Kirlian analysis in the modeling of non-equilibrium conditions with a gas electric discharge that simulates the primary atmosphere. Nano engineering, 12(30): 3-13 [in Russian].

Ignatov, I. \& Mosin, O.V. (2013c) Structural mathematical models describing water clusters. Journal of Mathematical Theory and Modeling, 3(11): 72-87.

Ignatov, I. \& Mosin, O.V. (2014) Color Coronal (Kirlian) Spectral Analysis in Modeling of Nonequilibrium Conditions with the Gas Electric Discharges, Simulating Primary Atmosphere, Biomedical Radioelectronics, 2: 42-51. [in Russian]

Inaba, H. (1988). Super-high sensitivity systems for detection and spectral analysis of ultraweak photon emission from biological cells and tissues. Experientia, 44: 550-559.

Kim, T.J (2002) Biophoton emission from fingernails and fingerprints of living human subjects. Acupuncture Electrother Res., 27:85.

Kirlian, S.D. (1949) Method for receiving photographic pictures of different types of objects. USSR Patent № 106401.

Kiang, J.G., Ives J.A. \& Jonas, W.B. (2005) External bioenergy-induced increases in intracellular free calcium concentrations are mediated by $\mathrm{Na}^{+} / \mathrm{Ca}^{2+}$ exchanger and L-type calcium channel. Mol. Cell Biochem., 271:51.

Kwan-Hoong, Ng. (2003) Non-ionizing radiations - sources, biological effects, emissions and exposures. Proceedings of the International Conference on Non-Ionizing Radiation at UNITEN (ICNIR2003). Electromagnetic Fields and Our Health. 20-22 October 2003.

Liboff, A.R., Williams, T., Strong, D.M. \& Wistar, R. (1984) Time-varying magnetic fields: effect on DNA synthesis. Science, 223: 818-820.

Lin, S., Chevalier, G., Lin, H., Ross, T. \& Lin, T. (2006). Measurement of biophoton emission with a single photon counting system. J. Altern. Complement. Med., 12:210.

Marino, A.A (Ed.) (1988) Modern Bioelectricity. Marieel Dekker, New York, Basel, ISBN 0-8247-7788-3.

Marinov, M. \& Ignatov, I. (2008) Color Kirlian spectral analysis. Color observation with visual analyzer. Euromedica, Hanover, 57-59.

Miller, M.W. (1986) Extremely low frequency (ELF) electric fields: experimental work on biological effects. CRC Handbook of biological effects of electromagnetic fields, 138-168.

Mosin, O.V. (2011) Magnetic devices for water treatment. C.O.K. Publishing House "Media Technology" (Moscow), 6: 24-27 [in Russian].

Mosin, O.V. (2012) Advanced technologies and equipment for magnetic water treatment (review). Water supply and sanitary technique, 8: 12-32 [in Russian].

Motohiro, T. (2004). Biophoton detection as a novel technique for cancer imaging. Cancer Science, 95(8): 656-661.

Niggli, H.J. (2014a) Biophotons: Ultraweak light impulses regulate life processes in aging. Journal of Gerontology \& Geriatric Research 3 (143) doi:10.4172/2167-7182.1000143.

Niggli, H.J. (2014 b) Ultraweak electromagnetic wave radiation as biophotonic signals to regulate life processes. Journal of Electrical \& Electronic System (in press).

Niggli, H. (1993). Artificial sunlight irradiation induces ultra weak photon emission in human skin fibroblasts, Journal of Photochemistry and Photobiology B: Biology, 18 (2-3): 281-285.

Niggli, H.J., Tudisco, S., Privitera, G., Applegate,, L.A., Scordino, A. \& Musumeci, F. (2005) Laser-Ultraviolet-A induced ultraweak photon emission in mammalian cells. J. Biomed. Opt. 10 (2).

Nikolaev, Y.A. (2000) Distant Interactions in Bacteria. Microbiology, 69(5): 497-503.

Pehek, J.O., Kyler, H.J \& Faust, D.L. (1976). Image modulating corona discharge photography, Science, 194(4262): 263-270.

Popp, F.A., Li, K. \& Gu, Q. (1992) Recent advances in biophoton research and its application, World scientific, $1-18$. 
Popp, F.A., Quao, G. \& Ke-Hsuen, L. (1994) Biophoton emission: experimental background and theoretical approaches, Modern physics Letters B., 8: 21-22.

Popp, F.A., Chang, J.J., Herzog, A., Yan, Z. \& Yan, Y. (2002) Evidence of non-classical (squeezed) light in biological systems. Physics Letters A., 293(1-2): 98-102.

Popp, F.A. (2005) Essential differences between coherent and non-coherent effects of photon emission from living organisms. In: X. Shen, R. van Wijk (eds). Biophotonics. New York: Springer, 124 p.

Porter, N.A. \& Wujek, D.G. (1988) Reactive Oxygen Species in Chemistry, Biology, and Medicine. In A. Quintanilha, Ed. New York, Plenum Press, pp. 55-79.

Rauhut, M.M. (1985) Chemiluminescence. In: M. Grayson (Ed). Kirk-Othmer Concise Encyclopedia of Chemical Technology (3rd ed). New York, John Wiley and Sons, ISBN 0-471-51700-3, 247 p.

Rattemeyer, M., Popp, F.A. \& Nagl, W. (1981) Evidence of photon emission from DNA in living systems, Nature Wissenshanften, 68(11): 572-573.

Ring, E.F.J. \& Hughes, H. (1986) Real time video thermography in recent developments in medical and physiological imaging. Suppl. Journal of Medical Engineering and Technology, 86-89.

Sessler, G.M. \& Gerhard-Multhaupt, R. (eds) (1998) Electrets. Laplacian Press, Morgan Hill, California, USA, ISBN 1-885540-07-8.

Seto, A., Kusaka, C., Nakazato, S. et al. (1992) Detection of extraordinary large bio-magnetic field strength from human hand. Acupuncture Electrother Res. Int. J., 17:75.

Shimizu H., Suzuki, Y. \& Okonogi, H. (1995) Biological effects of electromagnetic fields. Nippon Eiseigaki Zasshi., 50(6): 919-931.

Sisodia, M.L. (2007). Microwaves: introduction to circuits, devices and antennas. New Delhy, New Age International Ltd., ISBN 8122413382, 602.

Vladimirov, Y.A. (1996) Studies of antioxidants with chemiluminescence. In: Proceedings of the International Symposium on Natural Antioxidants. Molecular Mechanisms and Health Effects. L. Packer, M.G. Traber \& W. Xin (eds.). pp. 125-144.

Wikswo, J. \& Barach, J. (1980) An estimation of the steady magnetic field strength required to influence nerve condition. IEEE Trans. Bio-Med. Eng., 27: 722-723.

Young, R.E. \& Roper, C.F. (1976) Bioluminescent countershading in midwater animals: evidence from living squid, Science, 191(4231): 1046-1048.

Zhadin, M.N. (2001) Review of russian literature on biological action of DC and low-frequency AC magnetic fields. Bioelectromagnetics, 22: 27-45.

Zlatkevich, L. \& Kamal-Eldin, A. (2005) Analysis of Lipid Oxidation. In: A. Kamal-Eldin \& J. Pokorn (Eds.). New York, AOCS Publishing, 281. 
The IISTE is a pioneer in the Open-Access hosting service and academic event management. The aim of the firm is Accelerating Global Knowledge Sharing.

More information about the firm can be found on the homepage:

http://www.iiste.org

\section{CALL FOR JOURNAL PAPERS}

There are more than 30 peer-reviewed academic journals hosted under the hosting platform.

Prospective authors of journals can find the submission instruction on the following page: http://www.iiste.org/journals/ All the journals articles are available online to the readers all over the world without financial, legal, or technical barriers other than those inseparable from gaining access to the internet itself. Paper version of the journals is also available upon request of readers and authors.

\section{MORE RESOURCES}

Book publication information: http://www.iiste.org/book/

Recent conferences: http://www.iiste.org/conference/

\section{IISTE Knowledge Sharing Partners}

EBSCO, Index Copernicus, Ulrich's Periodicals Directory, JournalTOCS, PKP Open Archives Harvester, Bielefeld Academic Search Engine, Elektronische Zeitschriftenbibliothek EZB, Open J-Gate, OCLC WorldCat, Universe Digtial Library, NewJour, Google Scholar

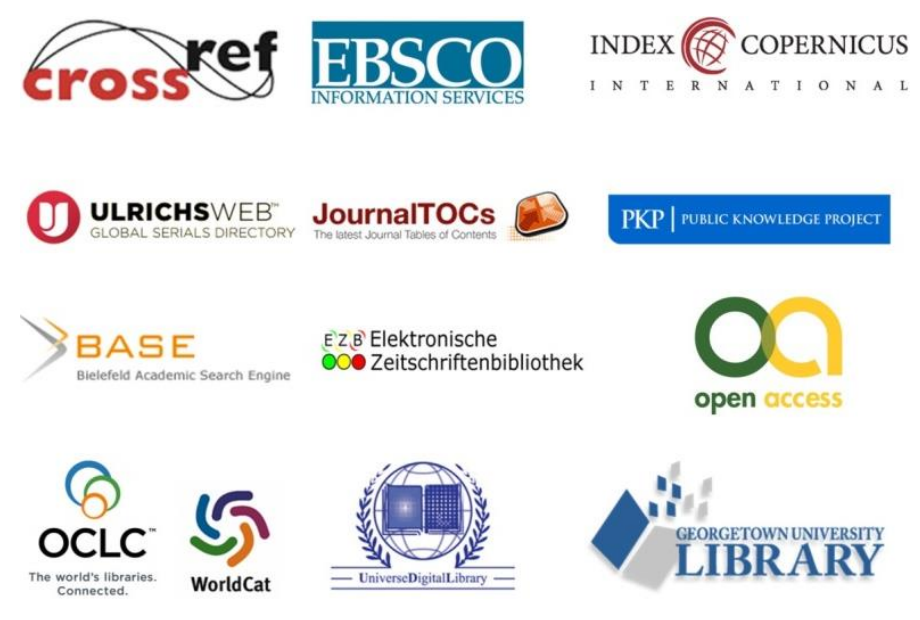

